\title{
LA VIDA UNIVERSITARIA EN LA EDAD ADULTA: RESPIRAR LA ARQUITECTURA
}

\author{
University life at adult age: Breathing the architecture
}

\section{Diana Amber ${ }^{1}$}

damber@ujaen.es

${ }^{1}$ Universidad de Jaén, España

Fecha de recepción: 6/03/2020

Fecha de aprobación: 7/04/2020

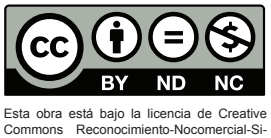

Esta obra está bajo la licencia de Creative
Commons Reconocimiento-Nocomercial-S nobraDerivada 4.0 Internacional.

ISSN (impreso): 2636-2139

ISSN (en línea): 2636-2147

Sitio web: https://revistas.isfodosu.edu.do/recie

\section{Resumen}

El aprendizaje a lo largo de la vida es promovido actualmente en Europa desde el sistema deEducación Superior en respuesta a la fluctuante sociedad contemporánea. En Portugal, siguiendo esta tendencia, desde 2006 se habilitaron pruebas de acceso a la universidad para mayores de 23 ańos para fomentar la igualdad de oportunidades. A pesar de que las potencialidades del estudiantado mayor son profusas, el índice de abandono de los estudios es alto. Por ello, este trabajo se propone como objetivo visibilizar estrategias de éxito reales, implementadas para la superación de las dificultades y el logro de metas académicas en el entorno universitario. Para ello, desde un enfoque cualitativo, mediante el uso de la entrevista en profundidad como instrumento de recogida de datos, se aborda un caso de éxito desde el marco de la investigación biográfico-narrativa. Los resultados muestran la trayectoria vital del caso, destacando motivaciones, estrategias $\mathrm{y}$ apoyos útiles para retomar y afrontar la formación superior en la edad adulta desde un enfoque introspectivo vivencial. Las conclusiones apuntan a la sistematización como clave para la conciliación familiar, académica y profesional, y al conocimiento previo del contexto, como principales estrategias del éxito académico. Se destaca que la edad y especialmente la experiencia de vida son recursos valiosos, no solo para la adaptación al cambio y el abordaje resiliente de situaciones complejas, sino también para la mejora de la enseñanza superior, desde los aportes de una visión reflexionada y madura e inserta en este contexto, por tanto, conocedora de sus entresijos.

Palabras clave: adultos, aprendizaje a lo largo de la vida, Educación Superior, igualdad de oportunidades educativas.

\section{Abstract}

The Higher Education System in Europe is currently promoting lifelong learning, as an answer for the fluctuating contemporary society. Following this trend, since 2006 in Portugal, access tests for people older than 23 years old were prepared, encouraging equal opportunities. Even though the potential of the older student body is profuse, the dropout rate is high. Therefore, the aim of this study is to raise awareness of success strategies implemented for overcoming difficulties and the achievement of academic goals in the university environment. To do this, from a qualitative approach, through the use of the in-depth interview as a data collection instrument, a case of success is presented from the biographical-narrative research framework. Results show the trajectory of the case, highlighting motivations, strategies, and useful supports to resume and face higher education in adulthood from an introspective-experiential approach. Conclusions indicate that systematization is key to family, academic and professional conciliation and to prior knowledge of the context, as the main strategies for achieving academic success. It should be noted that age and especially life experience, are valuable resources, not only for adaptation to change and resilient approach of complex situations, but also for higher education improvement, from the contributions of a thoughtful and mature vision and inserted in this context, thus, with awareness of hidden details.

Keywords: adults, lifelong learning, higher education, equal opportunity in education. 


\section{Justificación}

La creación del Espacio Europeo de Educación Superior ha supuesto fuertes cambios en la formación universitaria a nivel europeo, los cuales han contribuido a la adaptación del marco universitario a las nuevas demandas sociales promovidas principalmente por la fluctuación constante, el auge de la tecnología y la rápida caducidad de la información (Amber \& Martínez-Valdivia, 2018). Estas nuevas tendencias de la Educación Superior promueven la autonomía y actividad del alumnado y el desarrollo de competencias, especialmente de aquellas que permiten el aprendizaje a lo largo de la vida, tales como la capacidad de aprender a aprender (López et al., 2016; Martín, 2008). Esta competencia favorece la continuidad del aprendizaje en todas las facetas y momentos vitales, de manera que se propicie la adaptación de las personas al ritmo cambiante de la sociedad actual. Aunque no se trate de un concepto nuevo, pues la educación permanente ya fue promovida desde antańo como respuesta a la necesidad de actualización de las personas adultas (Delors, 1996), la educación a lo largo de la vida —o, como la denomina Rubio-Herráez (2007), "educación a lo largo y a lo ancho de la vida" - es un proceso continuo que no se limita a una edad o contexto determinado, sino que amplía sus horizontes incluyendo cualquier sistema de aprendizaje adquirido tanto por medios formales como no formales e informales (Amber \& Domingo, 2019).

En línea con lo anterior, a favor de la promoción del aprendizaje a lo largo de la vida y apostando por la igualdad de oportunidades, varios países de la Unión Europea, como Portugal, han incorporado modalidades de acceso a la universidad para acercar a nuevos públicos al mundo universitario y permitir que las personas que habían pausado sus estudios puedan retomarlos nuevamente.

En el caso de Portugal, desde el año 2006, con la publicación del Decreto-Lei n. ${ }^{o}$ 64/2006, de 21 de marzo, se comienzan a regular las pruebas destinadas a evaluar las capacidades de las personas de más de 23 años para incorporarse al sistema de Educación Superior. Asumiendo la lógica del aprendizaje a lo largo de la vida, este decreto permite flexibilizar el sistema de acceso a la universidad y, aunque atribuye la responsabilidad de la selección del alumnado adulto a las diferentes instituciones de Educación Superior portuguesas, prioriza como criterio la experiencia profesional de los candidatos. Esta iniciativa posibilita el acceso de perfiles personales, profesionales y académicos no tradicionales al entorno universitario.

Debido a que las pruebas de acceso para mayores de 23 años tienen la finalidad de evaluar la capacidad de los candidatos para desenvolverse con éxito en la universidad, el Decreto-Lei n. ${ }^{\circ}$ 64/2006 establece que la prueba debe integrar obligatoriamente tres elementos:

1. La valoración del currículo académico y profesional del candidato.

2. La evaluación de las motivaciones del candidato para acceder a la universidad (las cuales sugiere que pueden ser evaluadas a través de una entrevista personal).

3. La realización de pruebas teóricas y prácticas para evaluar los conocimientos y competencias necesarias para el ingreso y adecuado progreso en la titulación para la que solicite acceso.

Desde su publicación en 2006, el Decreto-Lei n. ${ }^{\circ}$ 64/2006 fue modificado años después por el Decreto-Lei n. ${ }^{\circ}$ 113/2014, del 16 de julio, y este, a su vez, por el Decreto-Lei n. ${ }^{\circ}$ 63/2016, del 13 de septiembre, sin alterar la información relativa a los componentes de evaluación ni al porcentaje de plazas disponibles que del cómputo total de estudiantes se reserva a los mayores de 23 años, debiendo ser este de al menos el $5 \%$ de las plazas disponibles.

Esta oportunidad relativamente reciente de acceso a la universidad permite que las personas que pueden llevar años alejadas del sistema educativo se integren en las aulas universitarias, enfrentándose a una nueva realidad, nuevos retos, dificultades y obstáculos, pero también esperanzas, ilusiones y expectativas. 


\section{AMBER \\ La vida universitaria en la edad adulta: respirar la arquitectura}

Esta medida ha incrementado notablemente la diversidad generacional presente en las aulas. La diversidad de edades permite la coexistencia, en una misma dinámica participativa y en un mismo espacio académico y social, de personas que participan de corrientes sociales y culturales propias de diferentes momentos históricos (Pachana et al., 2019). Hasta cinco generaciones diferentes, definidas por la literatura (Maldonado \& Osio, 2018), pueden encontrarse también en el ámbito universitario: los tradicionalistas, con menor presencia en la universidad, pues incluye a los nacidos antes de 1943; los baby boomers, cuya fecha de nacimiento se sitúa entre 1944 y 1960; la generación X, entre 1961 y 1980, caracterizada por la superación personal y profesional y el esfuerzo que ello requiere; los millennials (1981-2000), asociados a la autoestima y autoconfianza y por haber crecido durante la explosión digital; y la generación Z, los más jóvenes, nacidos a partir de 2001 y los que tienen mayor presencia en los primeros cursos de la Educación Superior. La diversidad generacional en el ámbito universitario supone que las personas con diferentes actitudes, valores, planteamientos vitales, etc., convivan e interactúen durante su proceso formativo en un mismo espacio y con las mismas metodologías de enseñanza, exigencias, recursos y sistemas de evaluación.

La universidad se ha abierto a diferentes públicos y ha creado un espacio de encuentro e intercambio entre perfiles de estudiantes muy diversos, no solo en cuanto a la edad, sino también las experiencias vitales, trayectorias, intereses, situaciones familiares, etc., aspectos condicionados en parte por la pertenencia a una determinada generación, pero también por las decisiones tomadas y las vivencias. Esta pluralidad genera un ambiente muy enriquecedor a nivel experiencial y de intercambio, y también de forma paralela, nuevas inquietudes, necesidades y retos que ha de superar la Educación Superior.

A pesar de la diversidad generacional que existe entre el propio alumnado de mayor edad en las universidades portuguesas (pues entre ellos se considera a todos los estudiantes que acceden con más de 23 años, incluyendo cuatro generaciones diferentes en este rango), el alumnado adulto manifiesta grandes potencialidades académicas motivadas principalmente por su mayor experiencia de vida y, en general, por una mayor voluntad para aprender, que contribuyen a su éxito académico (Munro, 2011). En líneas generales, sus motivaciones están relacionadas con el logro de objetivos profesionales y con la satisfacción mediante el aprendizaje, como señala Mangas et al. (2019). No obstante, pueden encontrar numerosos obstáculos en el camino, tales como la dificultad para conciliar los estudios con la vida profesional y familiar o la integración plena en el grupo de iguales, especialmente aquellos estudiantes de mayor edad (Quintas et al., 2014). Estas dificultades, entre otras, pueden empujar al alumnado adulto a desistir de su intento, lo que incrementa el porcentaje de abandono de los candidatos mayores de 23 años, como señala el estudio de Soares y Figueira (2011).

Sin embargo, existen casos de éxito que logran movilizar las estrategias necesarias para superar las dificultades al retomar los estudios tras años de separación del mundo académico. Su visibilización puede ayudar a inspirar a otras personas que aún no se hayan decidido a dar el paso de reiniciar los estudios. Desde este planteamiento, este artículo da a conocer cómo la experiencia de vida puede ser puesta al servicio de la superación personal y del logro de metas académicas desde un enfoque resiliente, a partir de vivencias personales reales. La resiliencia es entendida como la capacidad para adaptarse de forma positiva a situaciones adversas o estresantes (Uriarte, 2014). Esta capacidad en el marco universitario, según Vizoso-Gómez y Arias-Gundín (2018), es una característica esencial de aquellos estudiantes que, a pesar de enfrentarse a dificultades o situaciones de estrés, tienen un alto desempeño académico. Por tanto, siguiendo a Kristjánsson (2012), la resiliencia aparece asociada al éxito académico. El éxito es entendido en este trabajo como el logro del rendimiento académico necesario para aprobar las materias de la titulación, así como para la continuidad en el programa de estudios que permita la culminación de los mismos y la obtención del grado universitario en los tiempos establecidos por los planes de estudio (Martínez-González et al., 2003; Soria-Barreto \& Zúñiga-Jara, 2014). 
A partir de todo ello, se propone como objetivo principal de este trabajo la visibilización de estrategias de éxito implementadas en un caso real para la superación de las dificultades y el logro de metas académicas en el contexto universitario. De forma paralela, el estudio pretende evidenciar la apertura a nuevos perfiles que la modalidad de acceso para mayores de 23 ańos permite en las universidades portuguesas, y aportar claves para que la integración del estudiantado sea efectiva y real.

\section{Metodología}

Desde un abordaje cualitativo, inserto en el paradigma crítico, se busca la comprensión de los fenómenos tal como son vividos por los propios sujetos (Morgado, 2016). Por ello, se realiza un estudio de caso, profundizando en las lecturas que el sujeto hace de sus propias experiencias desde una perspectiva introspectiva vivencial. Siguiendo a Stake (2005), estas lecturas e interpretaciones del participante facilitan la comprensión de los puntos fuertes y débiles y posibilitan avanzar hacia la mejora. Por ello, dentro de los estudios de caso, se ha optado por la historia de vida (Arnal et al., 2001), desde un enfoque biográfico narrativo basado en la experiencia personal.

\subsection{Selección y descripción del caso de estudio}

Partiendo de la importancia del caso seleccionado (y de lo que este con su experiencia puede aportar) para la relevancia de este tipo de estudios narrativos (Domingo \& Bolívar, 2019), el informante que centra este trabajo se caracteriza por su experiencia universitaria como estudiante adulto, su disponibilidad para narrarla, sus habilidades comunicativas y por tratarse de un caso de éxito en este contexto. Además, posee una visión reflexiva y analítica basada en la metacognición de su propia trayectoria académico-profesional, que ha sido potenciada por su participación como ponente en una iniciativa universitaria de difusión del acceso a la universidad para mayores de 23 años.

El pseudónimo bajo el que se preserva la identidad del participante de este estudio es Peter, en honor al arquitecto Peter Zumthor, al que admira. Peter es un varón residente en Portugal que inició sus estudios a los 49 ańos de edad, por lo que se encuentra en la franja etaria de los denominados por la literatura "estudiantes adultos" (mature students) (Mallman \& Lee, 2016) y pertenece a la llamada generación X. Peter se encuentra en $2 .^{\circ}$ curso del grado universitario Mestrado integrado em Arquitetura de una prestigiosa universidad portuguesa. Actualmente compagina sus estudios con su vida profesional y con su vida familiar, y es esposo y padre de un hijo adolescente.

\subsection{Fuentes y proceso de recogida y análisis de la información}

Se analizan diferentes fuentes de información en torno al sujeto y a la realidad de los estudiantes mayores en la universidad de referencia. La principal fuente de información es el propio sujeto de estudio y los momentos e instrumentos de recogida de datos son diversos. La entrevista en profundidad es el principal instrumento utilizado, mediante esta se invita al sujeto a reconstruir su historia de vida. La información obtenida por este medio se complementa y matiza con conversaciones informales, intercambio de emails y mensajes a través de WhatsApp. Los intercambios de información con el participante se produjeron durante el mes de febrero de 2020 en la ciudad portuguesa en la que cursa sus estudios. El guion de la entrevista es semiestructurado, y permite la expresión libre del participante en torno a unas guías conversacionales flexibles relativas a su trayectoria académico-profesional y a su experiencia universitaria. Antes de su aplicación, esta entrevista es validada previamente por juicio de expertos. La información obtenida a través de la entrevista es procesada, redactada como relato de vida y validada por el propio informante, que asegura concordar íntegramente con lo expresado en el informe.

La información aportada por Peter es triangulada con otras fuentes tales como las entrevistas colaterales exploratorias realizadas al personal de apoyo al estudiante y a la coordinadora del "Núcleo de formación a lo largo de la vida" (NFLV).

A partir de todo el material recogido se procede a su categorización e identificación de hitos y puntos de 
inflexión para su análisis, conjugación e interpretación y a la posterior redacción del informe de resultados que se presenta en este estudio. El informe comienza con la definición del perfil académico-profesional de Peter, y posteriormente se centra en los hallazgos obtenidos en torno a seis dimensiones emergentes del discurso del participante. Estas dimensiones analíticas hacen referencia a sus motivaciones, entendidas como los motivos que dirigen la conducta (Soriano, 2001); a las dificultades encontradas y las estrategias de superación implementadas para afrontarlas; a los apoyos recibidos y potencialidades percibidas; a los aspectos más valorados de la experiencia universitaria; a las estrategias académicas empleadas para el estudio en condiciones de éxito y, por último, a los retos o desafíos que Peter lanza al sistema de Educación Superior.

\section{Resultados}

La arquitectura siempre atrajo el interés de Peter. En su juventud en Brasil, compaginaba su trabajo en la restauración con sus estudios universitarios de arquitectura. Una propuesta laboral realizada por uno de los huéspedes del hotel en el que trabajaba pospuso los estudios universitarios iniciados por Peter en Brasil, a tan solo un año y algunos meses de su comienzo. Fue así como llegó a Portugal como chef de cocina, con intención de vivir nuevas experiencias laborales y culturales y con la idea de volver en un tiempo a retomar sus estudios, tiempo que se extendió más de lo esperado.

La vida profesional y personal y las diferentes oportunidades laborales que le fueron surgiendo lo llevaron a trabajar en diferentes ciudades y países del mundo (Angola, China, etc.) gracias a su habilidad para la habilitación y adecuación de los espacios de trabajo, motivada por su propia experiencia en la cocina unida a su vocación y talento arquitectónico. Esta combinación de elementos le hizo promocionar en su trabajo, logrando articular dos ámbitos profesionales tan aparentemente distantes como la hostelería y la construcción.

Actualmente, Peter continúa amando "construir el espacio", como él lo denomina, y su profesión le permite expresarse en este sentido, pues gestiona tres empresas de construcción, dos de ellas en Angola y una en Portugal. Una de estas empresas mantiene la esencia de la mezcla profesional que ha definido la trayectoria de Peter, pues está orientada específicamente al diseño y construcción de locales de restauración, tales como bares, hoteles, etc.

\subsection{Análisis motivacional: "Siempre estuvo aquí dentro, adormecido"}

A los 49 años de edad decidió retomar sus estudios universitarios de arquitectura, pues lo que había comenzado como un paréntesis en su formación se había extendido durante demasiado tiempo. Fueron 25 años en los que Peter afirma que la arquitectura siempre estuvo dentro de él, adormecida, latente, pero dejándose entrever en su forma de enfocar el trabajo, en su mirada analítica de los espacios y los decorados... como su trayectoria profesional lo ha demostrado.

Tiene unas altas expectativas en relación con la formación universitaria. Quiere aprender para mejorar lo que ya hace profesionalmente, así como para conocer nuevas técnicas y descubrir nuevos materiales y tecnologías de construcción, pues considera que la investigación es una parte esencial del desarrollo profesional. No obstante, reconoce que la decisión de volver a estudiar fue importante en su vida, y requirió de una gran organización previa que le permitiese conciliar la vida personal y profesional con la académica, a la que dedica unas 8 horas diarias. Considera que la estabilidad es necesaria para poder retomar los estudios, estabilidad en tres sentidos: emocional, financiera y familiar. También afirma que, si cualquiera de estos aspectos falla, las posibilidades de éxito se reducen notoriamente.

Peter se impone a sí mismo altas exigencias personales, pues siente que, disponiendo de menos años de vida profesional para aplicar sus estudios de arquitectura que sus compañeros, debe aprovechar al máximo la formación, terminar lo antes posible y con los mejores resultados. Así, está dispuesto a sacrificar el plano social, limitándolo prácticamente a su familia, para dedicar ese tiempo a la lectura, al estudio y a la realización de trabajos académicos. 


\subsection{Dificultades y estrategias de superación: "Choque de edades"}

Peter asegura, sin dudar, que la primera dificultad que tuvo que superar en su adaptación a la vida universitaria fue el "choque de edades" con sus compañeros. Especialmente en cuanto a la responsabilidad, pues considera que la juventud en muchos casos no valora ni aprovecha la oportunidad que tiene de progresar y aprender, lo que choca con su forma de entender la formación. Sintetiza en su discurso esta idea con ayuda de una antítesis que muestra dos posturas opuestas frente a la vivencia de la formación: "Yo voy a clase para recibir la clase. Yo no voy a clase para divertirme, ni para...”. Estas sentidas diferencias intergeneracionales alcanzan su mayor exponente en el abordaje de trabajos grupales, método de trabajo del que no disfrutaba Peter, especialmente al inicio de sus estudios, hasta que pudo conformar un equipo de compańeros con el cual sentirse cómodo por compartir objetivos y valores similares, y de los que conoció a las familias (pues considera importante que la familia esté más implicada en la formación universitaria de los nuevos estudiantes).

La adecuación del horario fue otra de sus mayores dificultades, teniendo en cuenta la rigidez del horario de su titulación, que se imparte solo matinalmente, mientras que otras titulaciones ofrecen un turno nocturno que facilita la conciliación con las obligaciones familiares y profesionales. Esta dificultad, una de las más complejas de superar por los estudiantes adultos, también es destacada por la coordinadora del NFLV, que es consciente de que no todas las titulaciones ofrecen la flexibilidad horaria necesaria para conciliar vida personal, académica y profesional. Así, la universidad cambió el ritmo de vida de Peter, quien tuvo que amoldar el plano social, familiar y profesional en torno a ella, puesto que "el horario de las clases mandaba en todo, y manda aún hoy: manda en el trabajo, manda en la familia, en lo social... manda en todo". Sus ideas están claras, la culminación de sus estudios es su prioridad, por lo que en este momento relega todos los posibles proyectos familiares y profesionales y mantiene en claro su objetivo.
Peter piensa que la organización es elemental para afrontar un cambio de vida semejante, y que la disciplina y la sistematización son básicas para asegurar el éxito. En sus propias palabras: "Yo consigo trabajar, consigo estar con mi familia, consigo estar con mi hijo todos los días, consigo estar en la empresa todos los días, lo consigo, lo consigo: ¡organización!".

Además, apunta que el NFLV, inscrito en su Universidad, fue de gran ayuda, tanto para la superación de las pruebas de acceso como para el progresivo acercamiento a la comunidad universitaria. Así, valora todas las iniciativas y actividades que propone esta unidad para facilitar el acceso de los mayores de 23 años a la universidad, tales como cursos de matemáticas, de portugués, de inglés, de redacción del currículum, etc. Asevera que la participación en estas actividades, y el seguimiento de las orientaciones del personal de la unidad, le permitieron diferenciarse del resto de candidatos que optaban a las mismas plazas. Razones por las que la coordinadora del NFLV lo define como una persona "impecable", así como por su excelente disposición para colaborar en las iniciativas que requieran de la narración de su experiencia.

\subsection{Apoyos y potencialidades: "Con los pies en el suelo"}

Su mayor apoyo en todo este proceso es, sin duda, su familia: su mujer y su hijo, que lo han apoyado desde el inicio, han aceptado la nueva situación y se han adaptado a ella, facilitándole la posibilidad de retomar los estudios; de su narrativa se desprende un enorme agradecimiento y una verdadera devoción hacia ellos. Su entorno profesional también le ha facilitado el trámite, sus colegas y colaboradores le aportan la tranquilidad necesaria para dejar parte del trabajo en sus manos, pues no puede dedicarle tanto tiempo como antes. De este modo, le alegra poder decir que "la familia y la empresa me apoyaron al $100 \% ”$.

En la actualidad, Peter no recibe apoyo institucional, ni económico, ni de asesoría, aunque valora sobremanera y no olvida el trabajo y las recomendaciones aportadas por el NFLV en los momentos de preparación al acceso a la universidad. 
La experiencia dada por la edad es sentida por Peter como su mayor potencialidad para el aprendizaje. Vive la edad como una ventaja que le ayuda a no vacilar, a tener claras sus prioridades y los pasos que ha de dar para lograr sus metas, y asegura que "Yo sé lo que quiero, no tengo dudas, que la juventud aún tiene".

Su experiencia profesional en el ámbito de la construcción le permite ser conocedor de los métodos, estrategias y materiales que darán buen resultado y de aquellos que no funcionarán. Esto le facilita arriesgar, innovar, ir más allá en sus trabajos y presentaciones, pero siempre "con los pies en el suelo", partiendo del conocimiento y la competencia que le aporta su experiencia.

Además, gracias a su experiencia puede resolver las actividades prácticas con gran agilidad. Algunos de los trabajos que los docentes proponen son tareas que ya ha enfrentado en el mundo laboral, por lo que sabe de antemano cual será el resultado o el producto final. No obstante, no renuncia a la investigación previa y a la realización de todos los pasos que el docente propone y, además, en el producto final arriesga introduciendo algo nuevo que pueda ser evaluado por el docente y que suponga un avance para él.

\subsection{Valores de la experiencia universitaria: "Respiran la arquitectura"}

La universidad ha supuesto para Peter no solo grandes cambios, sacrificios y esfuerzo, sino también una gran satisfacción, sentimiento de autorrealización y aprendizajes para la vida. Destaca tres aspectos que aprecia profundamente. En primer lugar, sus compañeros más cercanos, con los que ha formado un grupo sólido, aquellos que define como "jóvenes espectaculares" que, como él, "respiran la arquitectura". Son jóvenes con los que se identifica por su pasión por la disciplina y su fuerte dedicación y cuyo objetivo es lograr siempre la calificación máxima. En palabras de Peter: "pensamos siempre en el 20", y añade que "10 es aprobado, pero es mediocre", estas palabras reflejan la excelencia que busca Peter en su quehacer diario.
Valora también la gran apertura del profesorado, que no se limita a la resolución de problemas y situaciones acontecidos en el aula, sino que además ofrece asesoramiento y consejo al alumnado que lo requiera. En tercer lugar, Peter asegura haber creado un "hábito de lectura masiva". Reconoce preferir los libros a internet, pues es consciente de que la información que muestra la red no tiene filtros y no siempre es exhaustiva, y los libros le merecen mayor confianza. Pasa horas en la biblioteca ampliando información y documentándose, invierte en material bibliográfico y ha formado una colección personal con aquellas obras de las que disfruta.

\subsection{Estrategias académicas: "Expandir el pensamiento"}

Su estrategia fundamental de estudio es una alta sistematización y estructuración de los tiempos y tareas. Se considera una persona sistemática, cualidad que admite haber adquirido durante su etapa de chef. Esta característica le facilita estudiar de lunes a jueves las materias teóricas, ampliando diariamente la información de lo visto en clase y revisando previamente, antes del inicio de las materias, lo que se explicó la semana anterior, y dedica los viernes, sábados y domingos a los trabajos prácticos. Todo ello, sin dejar de atender a su familia y sus obligaciones laborales. También estudia inglés, pues en tercer y cuarto curso tendrá materias impartidas íntegramente en este idioma y quiere estar preparado para ello.

Piensa que lo mejor para aprender es no ceñirse al mínimo, sino "expandir el pensamiento". No solo saber cómo se resuelve una tarea, no solo aplicar las fórmulas, sino también conocer por qué razón. Como estrategia docente, valora la estimulación del aprendizaje, no quedarse solo en lo tratado en clase, sino ampliar, reflexionar sobre cómo se ha llegado hasta ahí, y también sobre la evolución o impacto social que tendrá en el futuro cada uno de los proyectos que emprende. Por ello valora especialmente a aquellos docentes que reconocen que nadie tiene el conocimiento pleno y que proponen cuestiones para la incentivación de la investigación. 
Valora en un profesor que sea directo, que exprese claramente cuando no se está siguiendo el camino correcto en el aprendizaje, pues gusta enterarse de los errores para atajarlos lo antes posible. Las falsas esperanzas y los rodeos, para él, retrasan y perjudican el aprendizaje.

En cuanto al proceso evaluador, aprecia especialmente que el docente realice una evaluación continua y formativa a lo largo del curso ofreciendo retroalimentación sobre los trabajos y tareas entregadas, pues le permite conocer la evolución de su aprendizaje y encauzarlo correctamente. Demanda entregas continuadas, semanales, para estimular el aprendizaje y mantener activo el recuerdo de lo aprendido. Tiene una visión negativa de la evaluación final, que no facilita este proceso formativo continuado, y asegura que si el docente propone un sistema de evaluación sumativa final, "todo el mundo va a estudiar el día de antes”. Tampoco la coevaluación es una buena estrategia evaluativa según su experiencia y opinión. La evaluación entre iguales, asegura que es banal cuando gran parte de los compañeros que evalúan y opinan sobre el trabajo de los demás no tienen la preparación suficiente ni el interés hacia el contenido que evalúan. En sus palabras, "somos cuarenta: veinte aparecen cuando les apetece, después, de los otros veinte, cinco están ahí porque el padre los obliga...", así, considera que tan solo quince opiniones pueden aportarle algo para su aprendizaje y, por tanto, no piensa que sea una buena forma de rentabilizar el tiempo de estudio. La misma opinión, y por las mismas razones, le merecen las actividades de debate en el aula, de las que no critica la falta de estrategia docente en su implementación por parte del profesorado, sino la falta de estímulos para el aprendizaje que siente que se derivan de las mismas.

\subsection{Retos de la Educación Superior: "Un año 0"}

Uno de los aspectos que, según Peter, debería mejorar en relación con la atención del estudiantado adulto es la divulgación de la posibilidad de acceso para mayores de 23 ańos en las universidades portuguesas. Afirma que aún hoy, años después de la implementación de este sistema de acceso, pocas personas conocen esta posibilidad, según el propio Peter ha podido comprobar en las reacciones de sorpresa de las personas con las que comparte los relatos de su experiencia académica actual.

Aunque el primer paso sea incrementar la divulgación de la iniciativa, para Peter también es esencial conocer el lugar en el que se va a estudiar de forma previa al acceso. De hecho, él mismo visitó la Facultad de Arquitectura en múltiples ocasiones antes de iniciar sus estudios, a fin de dominar el espacio, conocer los transportes, las zonas, etc. En este sentido, propone la creación de un "año 0", entendido como un periodo de preparación antes del inicio de la formación universitaria, no necesariamente de 12 meses, sino de 6 o menos. Su meta sería el acercamiento del alumno a la realidad que enfrentará cuando comience, tanto a nivel organizativo como de exigencias académicas. Serían orientaciones que aporten información básica para el desenvolvimiento diario en las clases y que hagan ver a las personas que su vida va a cambiar si deciden dar este paso, y de qué modo y a qué nivel lo hará, de manera que puedan analizar o reflexionar si realmente tienen la disponibilidad, el apoyo familiar, el tiempo y la dedicación que la universidad demanda, evitando así la creación de situaciones de estrés, agobio y el consiguiente abandono de los estudios, algo bastante frecuente entre los alumnos que acceden por la convocatoria de acceso para mayores de 23, según comenta el personal del NFLV.

Su experiencia académica le hace pensar que en la universidad "el alumno es un número": el acceso a la biblioteca es electrónico, la mayoría de las gestiones se realizan a través de la plataforma, si un estudiante deja de asistir a clase se desconoce la razón, etc. Siente una deshumanización de los servicios universitarios, caracterizados por la frialdad y la digitalización de los procesos. Así, reclama un trato más cercano y humanizado y enfatiza que, excepcionalmente, sí ha encontrado este tratamiento cercano y acogedor en el NFLV, cuyo personal muestra una alta disponibilidad para hablar, orientar y atender al alumnado de forma individualizada. 


\section{Discusión y conclusiones}

La historia de vida de Peter es un claro ejemplo de éxito y adaptación resiliente al marco universitario, puesto que ha sido capaz de afrontar las dificultades encontradas durante su experiencia universitaria, integrándose con éxito académico en un nuevo entorno. Su trayectoria evoca el recuerdo de tantas personas que en su juventud tuvieron que relegar los estudios para acceder al mercado laboral y que continúan diariamente con el deseo insatisfecho de continuar formándose, aunque quizá no hayan tenido la oportunidad o el valor necesario para poder retomarlos. Así, la motivación principal hacia la formación universitaria de Peter es cubrir ese anhelo, cerrar esa etapa de la vida que dejó abierta. De forma paralela, reclama aprendizajes útiles para la vida profesional, aunque no buscando una oportunidad o mejora laboral, motivación principal presente en otros trabajos (Barros et al., 2019; Santos \& Nascimento, 2012), sino con la pretensión del perfeccionamiento profesional y la superación personal, propia de su generación.

Peter es un caso de superación de barreras, de progreso, adaptación y esfuerzo, desde una precisa organización y un enfoque sistemático que le ayuda a organizar su tiempo y hace posible conciliar las obligaciones profesionales y la dedicación a la familia con el alto rendimiento académico. De este modo, supera dificultades de conciliación presentes con frecuencia en los estudiantes adultos (de Almeida et al., 2016), que muchas veces pueden redundar en la desistencia del intento (Soares et al., 2010).

El "choque de edades" recogido también por la literatura como una de las problemáticas de integración grupal del estudiantado de mayor edad (Chapman, 2017; Quintas et al., 2014) también está claramente presente en el discurso de Peter, que percibe una diferencia abismal entre sus intereses y los de muchos otros compañeros de grupo. No obstante, franquea también esta barrera al lograr formar parte de un grupo de trabajo intergeneracional con el que se identifica por su amor hacia la arquitectura (usando la metáfora que da título a este trabajo) y a cuyos miembros aprecia y valora, hecho que subraya las potencialidades y virtudes de las relaciones intergeneracionales, posibilitadas en el marco universitario por la diversidad de edades que promueve la modalidad de acceso para mayores de 23 ańos.

La incertidumbre y duda que puede generar enfrentarse a un entorno desconocido, como lo es el marco universitario para la población que lleva tiempo al margen del sistema educativo, queda patente también en otros estudios que aseguran que los estudiantes adultos necesitan conocer el espacio de forma previa (Hammond, 2004). Esta incertidumbre puede verse acrecentada por la frialdad y la digitalización de muchos procesos académicos, especialmente los burocráticos, así como la ausencia de un trato personal por parte de muchos de los servicios universitarios, señalada por Peter. En respuesta a ello, dominar el espacio es uno de los consejos fundamentales derivados de esta historia de vida, útil como recomendación para aquellas personas que quieran embarcarse en estudios universitarios y también como propuesta de acción para aquellas instituciones que pretendan acercar al público a sus instalaciones y ofertas formativas, mediante el ofrecimiento de un curso preparatorio previo al ingreso en el centro, lo que Peter denomina "año 0".

Como se extrae de la narrativa de Peter, la edad supone un gran potencial para el estudiante, por la madurez, constancia y clarificación de ideas que aporta a quien la acumula. La experiencia dada por la edad permite, mediante estrategias metacognitivas (Osses \& Jaramillo, 2008) y una mayor autoeficacia (Barros et al., 2019), desde una mirada analítica y reflexiva, valorar las experiencias de aprendizaje que contribuyen favorablemente al progreso académico y profesional. Así, los estudiantes mayores se convierten en buenos analistas de los métodos de enseñanza, como afirman Badalo y César (2008), lo que les permite aportar claves, desde una visión reflexionada y madura, para la mejora de la Educación Superior.

Una de las principales críticas pedagógicas de Peter a los métodos docentes se dirige al proceso evaluador. Esto es debido a que el sistema de evaluación 
tradicionalmente predominante en la Educación Superior basado en una prueba única final, aún obsoleto según las corrientes pedagógicas promovidas desde el Espacio Europeo de Educación Superior (Martín et al., 2016), sigue presente en muchas aulas universitarias. Contra esto, su relato resalta las bondades de la evaluación continua y formativa, como motor de aprendizaje y clave para la asimilación y afianzamiento progresivo de conocimientos y la adquisición de competencias. Además, en línea con los planteamientos apoyados en la ubicuidad del aprendizaje (Burbules, 2012) y buscando la profundización en el contenido de estudio, el relato de Peter muestra que el aprendizaje no queda circunscrito al entorno universitario y que el profesorado puede y debe ayudar al alumnado a descubrir este proceso ubicuo de expansión progresiva del aprendizaje y a potenciar su autonomía. En línea con la anterior, Peter considera la expansión del conocimiento, más allá de lo aprendido en el aula, como una de las estrategias eficaces que le permiten un alto rendimiento académico. No obstante, el anhelo de profundización de Peter es contrario a los resultados mostrados por los participantes de otros estudios como el de Monteiro et al. (2015), que reconocen adoptar un abordaje más superficial de los contenidos en su proceso de aprendizaje.

En definitiva, a pesar de las limitaciones propias de los estudios biográfico-narrativos, que dificultan su generalización por su naturaleza concreta, contextual y comprensiva, esta investigación da crédito de los logros y avances de la Educación Superior en inclusión, pero también del camino que queda por recorrer, a nivel institucional y social, para la correcta integración de nuevos perfiles en las aulas universitarias. Las actuales oportunidades de acceso a la universidad, que abren puertas a nuevos usuarios ampliando la diversidad generacional de las aulas, son un paso importante para la recepción y acogida de los estudiantes no convencionales. Sin embargo, queda mucho por hacer a nivel institucional para la facilitación de la integración de estas personas en el contexto universitario, temas pendientes tales como la mejora de la conciliación familiar, el acercamiento a los espacios, los métodos y procesos universitarios, etc., que pueden repercutir negativamente en el aprendizaje, la permanencia y la culminación de los estudios y que, actualmente, son carencias que tan solo pueden ser compensadas por la actitud resiliente y el esfuerzo realizado por los propios sujetos.

En conclusión, la historia de Peter no solo descubre en primera persona las dudas y factores adversos que los estudiantes adultos se pueden encontrar en el camino, sino también la forma en que han sido enfrentados con éxito. Su experiencia en este contexto, así como su visión reflexionada, madura e impregnada de vivencias universitarias en primera persona, puede ser útil tanto para otras personas que pretendan acceder a laEducación Superior en la edad adulta como para las instituciones universitarias, que pueden tomar pistas y claves de actuación para acercar al estudiantado no tradicional a la formación superior, facilitando su completa integración.

\section{Agradecimientos y reconocimientos}

Un especial agradecimiento al informante que, bajo el pseudónimo "Peter", ha puesto su interesante historia de vida a disposición de este trabajo, confiando a la autora el relato de sus experiencias y vivencias.

Una mención especial a la Red Iberoamericana de Investigación para el Desarrollo de la Identidad Profesional Docente (Red RIDIPD), de la que la autora forma parte.

Estudio financiado por el Ministerio de Educación, Cultura y Deporte (España) en el marco del Programa Estatal de Promoción del Talento y su Empleabilidad en I+D+i, Subprograma Estatal de Movilidad, del Plan Estatal de I+D+I.

\section{Referencias}

Amber, D., \& Domingo, J. (2019). Empleo y Aprendizaje Permanente en España: implicaciones en la formación de las personas adultas. Curriculo sem Fronteiras, 19(3), 1225-1242.

http://dx.doi.org/10.35786/1645-1384.v19.n3.25 
Amber, D., \& Martínez-Valdivia, E. (2018). La formación en Educación Superior. Retos y propuestas en docencia universitaria. Profesorado. Revista de Currículum y Formación del Profesorado, 22(3), 1-8. https://cutt.ly/Ctt9qWL

Arnal, J., Del Rincón, D., \& La Torre, A. (2001). Investigación educativa: fundamentos y metodología. Labor.

Badalo, C., \& César, M. (2008). Traçando o perfil dos estudantes adultos para compreender as suas características e responder às suas necessidades educativas: Quatro casos de estudo. International Journal of Developmental and Educational Psychology, 1, 399408. https://cutt.ly/xtW4KY0

Barros, R., Monteiro, A., \& Sousa, C. (2019). Autoeficácia formativa e envolvimento nos processos de aprendizagem de estudantes portugueses maiores de 23 anos. Revista Portuguesa de Educação, 32(1), 140 154. https://cutt.ly/jtt9ojp

Burbules, N. C. (2012). El aprendizaje ubicuo y el futuro de la enseñanza. Encounters/Encuentros/Rencontres on Education, 13, 3-14.

https://cutt.ly/Ltt9aMp

Chapman, A. (2017). Using the assessment process to overcome Imposter Syndrome in mature students. Journal of Further and Higher Education, 41(2), 112-119.

https://doi.org/10.1080/0309877X.2015.1062851

De Almeida, A. C., Quintas, H., \& Gonçalves, T. I. (2016). Estudantes não-tradicionais no ensino superior: barreiras à aprendizagem e na inserção profissional. Laplage em Revista, 2(1), 97-111.

https://doi.org/10.24115/S2446-6220201621122p.97-111

Delors, J. (1996). Informe de la UNESCO. La educación encierra un tesoro. Santillana.

Domingo, J., \& Bolívar, A. (2019). La investigación (auto)biográfica en educación. Octaedro.

López, B. G., Campos, C., \& Cerveró, G. A. (2016). Aprender a aprender en la universidad. Efectos de una materia instrumental sobre las estrategias de aprendizaje y el rendimiento académico. Cultura y Educación, 28(4), 790-810.

Hammond, C. (2004). Impacts of lifelong learning upon emotional resilience, psychological and mental health: Fieldwork evidence. Oxford Review of Education, 30(4), 551-568.

https://doi.org/10.1080/0305498042000303008

Kristjánsson, K. (2012). Positive psychology and positive education: Old wine in new bottles? Educational Psychologist, 47, 86-105.

https://doi.org/10.1080/00461520.2011.610678

Mallman, M., \& Lee, H. (2016). Stigmatised learners: mature-age students negotiating university culture. British Journal of Sociology of Education, 37(5), 684-701.

https://doi.org/10.1080/01425692.2014.973017

Mangas, C., Lopes, S., Ferreira, P., \& Beato, I. (2019). Accessing higher education after 23 years old: opportunities and motivations. Cadernos de Pesquisa, 49(172), 36-52.

https://doi.org/10.1590/198053145943

Maldonado, L., \& Osio, L. (2018). Diversidad generacional y la era del acceso: un reto para la gestión de gente. Visión gerencial, 17(1), 84-98.

https://cutt.ly/itW5sK2

Martín, E. (2008). Aprender a aprender: clave para el aprendizaje a lo largo de la vida. Tribuna Abierta. CEE Participación Educativa, 9, 72-78.

https://cutt.ly/EtW5g0U

Martín, S. Jiménez, N., \& Sánchez-Beato, E. (2016). La evaluación del alumnado universitario en el Espacio Europeo de Educación Superior. Aula Abierta, 44(1), 7-14.

https://doi.org/10.1016/j.aula.2015.03.003

Martínez-González, A., Martínez-Franco, A. I., Ponce-Rosas, R., \& Gil-Miguel, M. (2003). Perfil del estudiante de posgrado con éxito académico en la UNAM. Revista de Investigación e Innovación Educativa, (32). https://cutt.ly/gtYo4kQ

Morgado, J. C. (2016). O estudio de caso na 
investigação em educação. De Facto Editores.

Munro, L. (2011). 'Go boldly, dream large!': The challenges confrontingnon-traditional studentsat university. Australian Journal of Education, 55(2), 115-131. https://doi.org/10.1177/000494411105500203

Osses Bustingorry, S., \& Jaramillo Mora, S. (2008). Metacognición: un camino para aprender a aprender. Estudios pedagógicos, 34(1), 187-197. http://dx.doi.org/10.4067/S0718-07052008000100011

Panchana, M., Peña, H., Cochea, H., \& Reyes, A. (2019). La diversidad generacional y la micro-empresa familiar. Universidad Ciencia y Tecnología, 23(94), 10-10. https://cutt.ly/ptW5n6D

Quintas, H., Gonçalves, T., Ribeiro, C. M., Monteiro, R., Fragoso, A., Bago, J., Santos, L. \& Fonseca, H. M. (2014). Estudantes adultos no Ensino Superior: O que os motiva e o que os desafia no regresso à vida académica. Revista Portuguesa de Educação, 27(2), 33-56. https://cutt.ly/ytt9YT9

Rubio-Herráez, E. (2007). Aprendizaje a lo largo de la vida. Vivir y trabajar en una Europa del conocimiento. CEE Participación Educativa, 6, 14-29. https://cutt.ly/KtErB6N

Santos R., \& Nascimento, I. (2012). Estudantes maiores de 23 anos em psicologia: Motivaçôes e processo de integração. Revista Brasileira de Orientação Profissional, 13(1), 37-47. https://cutt.ly/Stt9OjS
Soares, D. L., Almeida, L. S., \& Ferreira, J. A. G. (2010). Percursos vocacionais e vivências académicas: $\mathrm{O}$ caso dos alunos maiores de 23 anos. Psicologia, educação e cultura, 14(1), 203-214.

https://cutt.ly/ptt9GVP

Soares, J., \& Figueira, M. (2011). Os novos públicos na Universidade de Lisboa: O seu retrato em 2011. Universidade de Lisboa.

Soria-Barreto, K., \& Zúńiga-Jara, S. (2014). Aspectos determinantes del éxito académico de estudiantes universitarios. Formación universitaria, 7(5), 41-50. http://dx.doi.org/10.4067/S0718-50062014000500006

Soriano, M. M. (2001). La motivación, pilar básico de todo tipo de esfuerzo. Revista de relaciones laborales, 9, 163-184.

Stake, R. E. (2005). Qualitative Case Studies. En N. K. Denzin \& Y. S. Lincoln (Eds.), The Sage handbook of qualitative research (pp. 443-466). Sage Publications Ltd.

Uriarte, J. D. (2014). Resiliencia y envejecimiento. European Journal of Investigation in Health, Psychology and Education, 4, 67-77.

https://doi.org/10.1989/ejihpe.v4i2.54

Vizoso-Gómez, C., \& Arias-Gundín, O. (2018). Resiliencia, optimismo y burnout académico en estudiantes universitarios. European Journal of Education and Psychology, 11(1), 47-59.

https://doi.org/10.30552/ejep.v11i1.185 